\title{
Functional Organization of Macaque V3 for Stereoscopic Depth
}

\author{
DANIEL L. ADAMS AND SEMIR ZEKI \\ Wellcome Department of Cognitive Neurology, University College London, London WC1E 6BT, United Kingdom
}

Received 30 October 2000; accepted in final form 18 July 2001

\begin{abstract}
Adams, Daniel L. and Semir Zeki. Functional organization of macaque V3 for stereoscopic depth. J Neurophysiol 86: 2195-2203, 2001. Recordings were made from single and small groups of cells in prestriate area V3 of the visual cortex of the Cynomolgus macaque (Macaca fascicularis). The majority of cells in V3 were selective for orientation and stereoscopic depth, these cells being segregated into two sets of functionally distinct columns. Orientation columns in V3 have been previously demonstrated; here we show that V3 also contains columns of segregated disparity-selective cells. On the basis of its cellular properties, functional organization, and intra-cortical connections, we propose that V3 contributes to the processing of stereoscopic depth information and that the parietal areas to which it projects use this information for the analysis of object depth and three-dimensional form.
\end{abstract}

\section{N T R O D U C T I O N}

Among the least studied of the cortical visual areas is V3, which lies deep within the lunate and inferior-occipital sulci in the macaque. The paucity of studies on V3 is perhaps surprising considering that it appears to occupy an "early" stage of visual processing and was among the first visual areas to be identified. V3 was discovered by virtue of its direct topographic projection from V1 (Cragg 1969; Zeki 1969) which originates in layer 4b (Burkhalter et al. 1986; Lund et al. 1975).

The posterior border of $\mathrm{V} 3$ lies at the representation of the horizontal meridian of $\mathrm{V} 2$ and $\mathrm{V} 3$. The anterior extent of dorsal $\mathrm{V} 3$ borders area $\mathrm{V} 3 \mathrm{~A}$ at the vertical meridian representation of these areas. Because parts of visual areas that represent the vertical meridian in each hemisphere are connected via the corpus callosum (Whitteridge 1965), V3's border with V3A can be visualized by staining for degenerated fibers after section of the corpus callosum (Van Essen and Zeki 1978; Zeki 1978a; Zeki and Sandeman 1976). Both V3 and V3A are organized retinotopically (Cragg 1969; Perkel et al. 1986; Zeki 1969, 1980). V3 consists of two portions: the dorsal portion of V3 (V3d) contains a representation of the lower contralateral quadrant of the visual field and ventral V3 (V3v) contains the upper contralateral quadrant (Gattass et al. 1988; Lyon and Kaas 2001).

Both V4 and V5 receive a forward projection from V3 (Zeki and Shipp 1988). Projections have been found directly from V3 and indirectly via V3A to the posterior parietal lobule (PPL) (Adams 1997). The stronger of the projections to the PPL is to cortex within the confines of the dorsal prelunate area (DP) (Andersen et al. 1990) and the caudal intraparietal area (CIP)

Present address and address for reprint requests: D. L. Adams, Dept. of Ophthalmology, University of California, 10 Koret Way, San Francisco, CA 94143-0730 (E-mail: dadams@itsa.ucsf.edu).
(Endo et al. 2000; Kusunoki et al. 1993; Sakata et al. 1997). In addition, the lateral intraparietal area (LIP) has asymmetric connections with V3 and V3A (Blatt et al. 1990). The pattern of connections to parietal (DP, CIP and LIP) and temporal (V4) areas places $\mathrm{V} 3$ in an hierarchically intermediate position between the V4 and V5 complexes.

$\mathrm{V} 3$, in common with $\mathrm{V} 3 \mathrm{~A}$, contains a predominance of orientation-selective cells (Baizer 1982; Burkhalter et al. 1986; Felleman and Van Essen 1987; Zeki 1978a,b). V3 cells with similar orientation preference are grouped into columns (Zeki 1978a). A similar functional architecture has been demonstrated in V5, where the property of direction selectivity is organized in a columnar fashion (Albright et al. 1984; Zeki 1974a). The grouping of cells into columns occurs in many areas of the neocortex. A clue to the functional tasks executed by any given area is afforded by the types of columnar systems it contains (Mountcastle 1997). The prevalence of orientationselective cells in V3, their preference for moving stimuli, and their grouping into columns have led some to identify it as an area specialized for the processing of dynamic form (Gegenfurtner et al. 1997; Zeki and Shipp 1988). Given the close connection between orientation selectivity and stereoscopic disparity tuning (Hubel and Wiesel 1970), we wondered whether there was any systematic relationship in the representation of the two within V3.

The horizontal separation of the eyes causes a slightly different image to fall onto each retina. The brain gains knowledge about depth by analyzing the disparity between the two retinal images. The brain's capacity to perceive depth from retinal disparity is called stereopsis. Cells sensitive to disparity have been found in most visual areas and have been classified according to the shape of their disparity tuning curve. Disparity-selective cells fall into two groups: tuned and asymmetric (Poggio and Fischer 1977; Poggio et al. 1988). Tuned cells respond with a narrow profile at a particular disparity (usually within $\pm 1^{\circ}$ of the fixation plane) and asymmetric cells respond over a broad range of disparities either in front of or behind the fixation plane.

Many V3 cells have strong binocular interactions, i.e., their binocular response are greatly facilitated or attenuated compared with the sum of their monocular responses (Zeki 1978a,b). Many V3 cells are also disparity-selective (Felleman and Van Essen 1987; Poggio et al. 1988). We report that the disparity-selective cells of V3 are organized into columns of similar cell type. The organization of orientation- and dispar-

\footnotetext{
The costs of publication of this article were defrayed in part by the payment of page charges. The article must therefore be hereby marked "advertisement" in accordance with 18 U.S.C. Section 1734 solely to indicate this fact.
} 
ity-selective cells in V3 leads us to propose that this area participates in the analysis of three-dimensional form.

\section{METHODS}

Thirteen Cynomolgus macaques (Macaca fascicularis) weighing between 1.5 and $2.0 \mathrm{~kg}$ were used. Monkeys were initially anesthetized with ketamine (Vetalar, Parke-Davis; $25 \mathrm{mg} / \mathrm{kg} \mathrm{im}$ ), intubated, and anesthetized by continuous intravenous infusion of propofol (Diprivan, Zeneca; $4-12 \mathrm{mg} \cdot \mathrm{kg}^{-1} \cdot \mathrm{h}^{-1}$ iv) and sufentanil citrate (Janssen; 3-8 $\mu \mathrm{g} \cdot \mathrm{kg}^{-1} \cdot \mathrm{h}^{-1}$ iv). A 5-mm craniotomy was made over the lunate sulcus and the dura reflected. A cylindrical plastic recording chamber was then bonded to the skull with dental cement and filled with 2\% agar-in-saline. After surgery, a neuromuscular blocking agent, pancuronium bromide (Pavulon, Organon Teknika; $0.4 \mathrm{mg}$ • $\mathrm{kg}^{-1} \cdot \mathrm{h}^{-1} \mathrm{iv}$ ), was infused to prevent eye movements. Streak retinoscopy was used to select appropriate lenses to focus the eyes onto a tangent screen $114 \mathrm{~cm}$ from the animal. Plano contact lenses were inserted to prevent corneal drying. The foveae and some distinctive retinal vascular landmarks were plotted onto the screen with a reversible ophthalmoscope and monitored regularly to track any residual eye drift. Recordings were made with glass-coated platinum-plated tungsten microelectrodes (Merrill and Ainsworth 1972) with an exposed tip length of 10-12 $\mu \mathrm{m}$. Spikes were sorted by amplitude using a window discriminator (Neurolog), digitized, and recorded by a computer at 20 -ms binwidth. Signals from the stimulus computer were integrated with the spike data and receptive field location to produce on-line poststimulus time histograms and to calculate average spike rates for each trial. During recording, animals were maintained under anesthetic and ventilated while their electrocardiogram (ECG), temperature, and end-tidal $\mathrm{CO}_{2}$ were monitored. All procedures were done in accordance with British Home Office regulations under the Animals (Scientific Procedures) Act 1986.

Multiple electrode penetrations were made over a period of 4-5 days. The reconstruction of electrode tracks from horizontal sections was aided by placing small electrolytic lesions at recording sites by passing current through the electrode ( $7 \mu \mathrm{A}$ for $7 \mathrm{~s}$, tip positive). Immediately after the experiment, the monkey was killed with an overdose of pentobarbitone sodium and perfused transcardially with warm saline, followed by 31 of $0.1 \mathrm{M} \mathrm{PO}_{4}$ buffered $4 \%$ paraformaldehyde. Buffered sucrose solution was then run through sequentially at 10,20 , and $30 \%$ concentrations. The brain was removed and placed in $0.1 \mathrm{M} \mathrm{PO}_{4}$ buffered $30 \%$ sucrose solution and kept at $4^{\circ} \mathrm{C}$ until no longer buoyant (2-4 days). Sections were cut horizontally at $60 \mu \mathrm{m}$ on a freezing microtome. Every section was saved and stored at $4^{\circ} \mathrm{C}$ in $0.1 \mathrm{M}$ phosphate buffer prior to histological reaction. Adjacent sections were stained for cytochrome oxidase (Wong-Riley 1979), myelin (Gallyas 1979), and Nissl.

\section{Stimuli}

Stimuli consisted of moving or stationary luminance contrast bars presented on an 18-in RGB computer monitor (Grundig), refresh rate $60 \mathrm{~Hz}$, positioned $114 \mathrm{~cm}$ from the animal. The orientation, size, shape, and color of the computer-generated stimuli could be changed to best suit the cell being studied. Receptive fields were plotted manually by moving a bar stimulus on the screen with a track ball. Both receptive fields of a binocular cell were plotted while occluding each eye in turn. Four boundaries were plotted for each receptive field to form two rectangular minimum response fields. Once receptive fields were digitized, computer-controlled trials were presented to discover the cell's most effective stimulus orientation, speed, color, size, and aspect ratio. Generally, $4-10$ presentations of each stimulus were necessary to produce a clean spike histogram, depending on the firing characteristics of the cell.

An adaption of the method used first by Henry et al. (1969) was employed to stimulate cells at different disparities. During paralysis, receptive fields became arbitrarily separated on the tangent screen because fusion was disrupted. A pair of optimal stimulus bars with a constant separation was swept over the screen so that each bar passed through one of the monocular receptive fields. Changing the stimulus bars' horizontal separation relative to the receptive field separation changed the disparity of the stimulus. Typically, cells were stimulated over a broad range of disparity $\left(\sim 5^{\circ}\right)$ in steps as small as 3 min of arc (1 $\mathrm{mm}$ on the tangent screen). This method of presenting simultaneously two monocular stimuli removed the need to superimpose the receptive field pairs with prisms. A limitation to studying disparity tuning in the anesthetized paralyzed preparation is that the eyes assume a divergent, drifting gaze, and a correction has to be used to stimulate corresponding points on each retina. We used the angular separation of the fovea's on the tangent screen as a measure of the angle between corresponding points in the two retinas. To correct our disparities for divergence and drift and calculate the position of the fixation plane, eye position was monitored between each recorded cell by projecting retinal vascular landmarks onto the tangent screen using a reversible ophthalmoscope. We found the reversible ophthalmoscope to be accurate to $\pm 1.0^{\circ}$ when plotting the positions of clearly visible vascular features of the retina, and this inaccuracy was reflected in our calculations of fixation plane position.

\section{Quantitative assessment of responses}

As well as qualitative assessment at the time of recording, we applied a quantitative method to judge cell selectivities. An index was calculated for each cell's responses to orientation, direction and color stimulus properties. Cells with index values $>0.5$ were considered biased and those with index values $>0.7$ were considered selective for the property in question.

Orientation index (OI) was calculated by the following formula

$$
\text { OI }=1-\frac{\text { Response to orthogonal orientation-Baseline }}{\text { Response to optimum orientation-Baseline }}
$$

where baseline is the background firing rate of the cell when no stimulus is present. We applied similar indexes to quantify our judgment of direction (DI) and color (CI) selectivity.

$$
\begin{gathered}
\text { DI }=1-\frac{\text { Response to opposite direction-Baseline }}{\text { Response to optimum direction-Baseline }} \\
\text { CI }=1-\frac{\text { Response to least effective bar color-Baseline }}{\text { Response to most effective bar color-Baseline }}
\end{gathered}
$$

We experimented with a similar disparity tuning index but found it unsatisfactory. Many cortical cells exhibit binocular facilitation, but the fact that binocular is more effective than monocular stimulation for a particular cell does not necessarily mean that the cell is disparity selective. Binocular facilitation may be independent of disparity tuning. We therefore only considered a cell disparity selective if its responses were sharply modulated over a small range of disparity. More specifically, only cells with half-width at half-height $<1^{\circ}$ in their disparity tuning curves were classified as disparity selective.

\section{RES U L T S}

The areal assignment of cells was done on the basis of sulcal topography and the locations of borders in brain sections from previous anatomical mapping studies. To visualize the V3/ V3A border, sections from brains where the splenium of the corpus callosum had been cut and the brain stained for degeneration were consulted (Van Essen and Zeki 1978; Zeki 1978a). Maps derived from V1 lesion studies (Cragg 1969; Zeki 1969) were used to locate the V2/V3 border. Receptive field sizes were also used to distinguish between V2 and V3, 
although they could not be used to differentiate V3 and V3A, whose cells have similarly sized RFs (Zeki 1978a). We could therefore be confident that cells were in V3 rather than V2 but not always as certain that they were in V3 rather than V3A.

A total of 225 cells was recorded from V3, 100 of which were tested for their selectivity for horizontal disparity. All of the units had their receptive fields within $20^{\circ}$ of the fovea and the majority were within $10^{\circ}$. Since we restricted our recording sites to the region of $\mathrm{V} 3$ at the fundus and deep anterior bank of the lunate sulcus (V3d), most receptive fields were restricted to the horizontal meridian and lower visual field (see Fig. 1A). However, a few V3 cells' field centers represent the ipsilateral visual field, a finding also demonstrated with fMRI in human
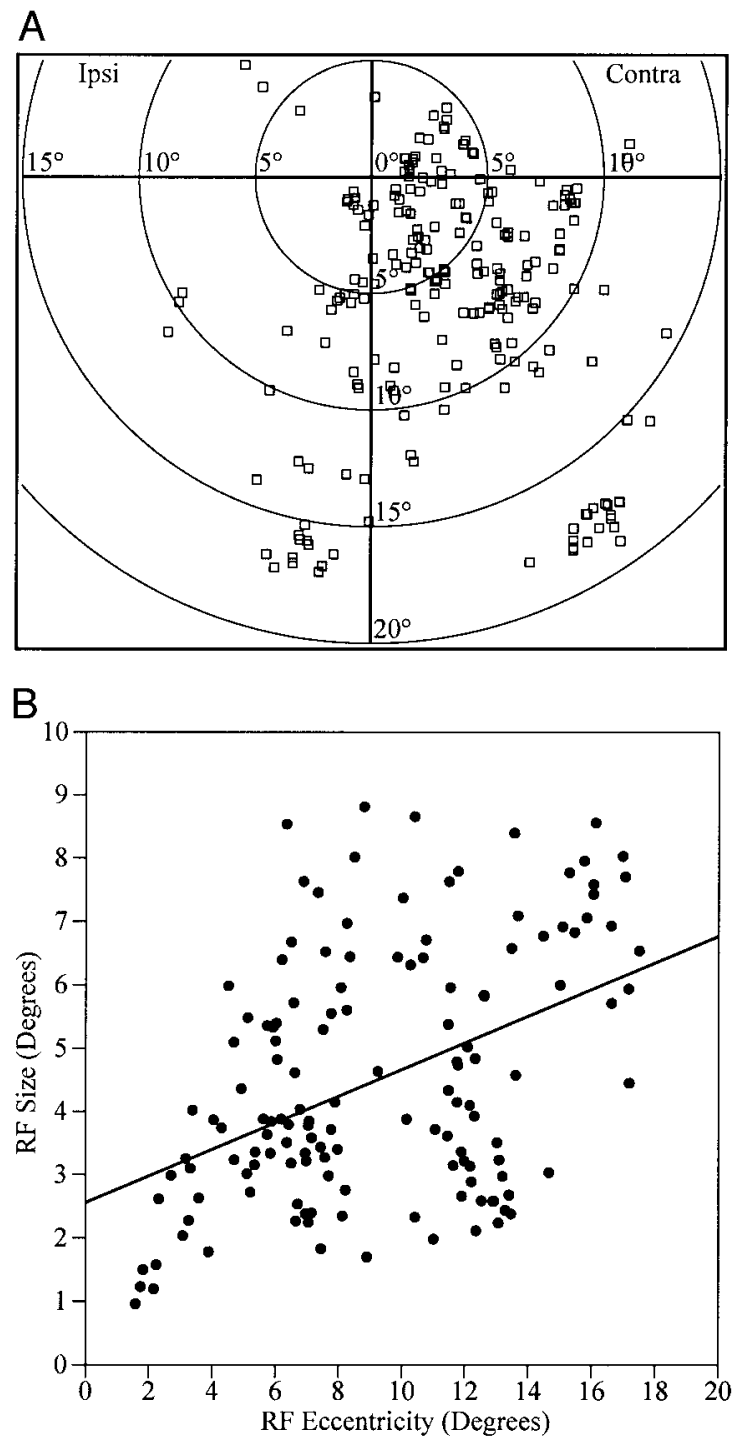

FIG. 1. A: receptive field center positions for the population of V3d cells recorded from in this study. Each data point is the mean position of the left and right eye receptive field centers in degrees for a single binocular cell. Fields are restricted to the central $20^{\circ}$ and, for the most part, to the inferior visual hemisphere and contralateral quadrant. $B$ : correlation of receptive field size with eccentricity. Size was calculated as the square root of the product of rectangular receptive field length and width. Each data point is the mean of both left and right receptive fields sizes for a single cell (in degrees) plotted against the mean eccentricity of the left and right receptive field centers in degrees. A linear regression is fitted to the data.

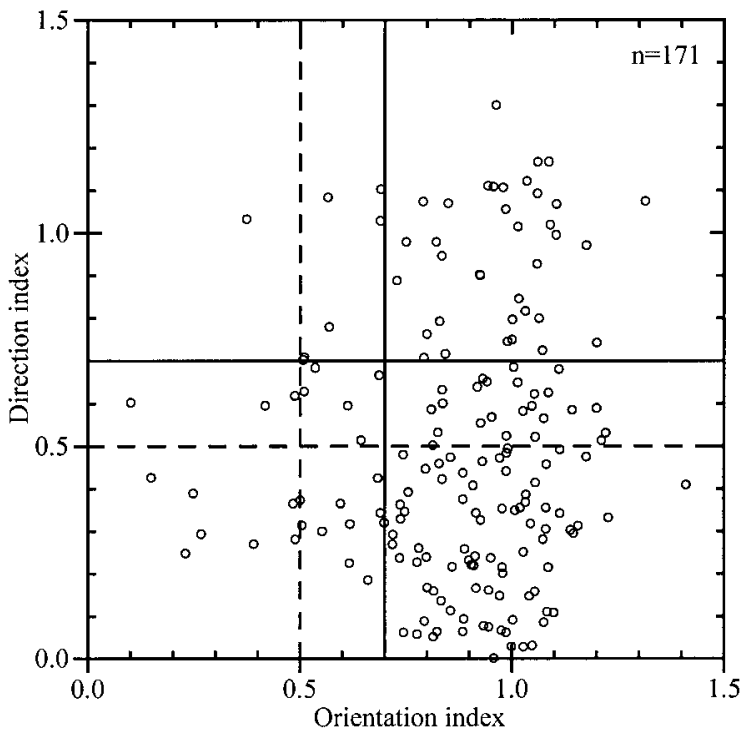

FIG. 2. Scatter plot of orientation and direction indexes (see METHODS for their formulae). Values of either index $>0.5$ (- - ) indicate a bias, and values $>0.7$ ( - ) indicate selectivity. The majority of V3d cells presented here are orientation selective, some are both orientation and direction selective, but very few are direction but not orientation selective.

V3 complex (Tootell et al. 1998). Figure $1 B$ shows how receptive field size varied as a function of eccentricity.

\section{Orientation/direction selectivity}

We confirmed that orientation selectivity was a characteristic property of V3 cells; the majority (85\%) was orientation selective as qualitatively judged by the experimenter. We calculated orientation indices (OI) for our population of V3 cells to quantify these judgments (see METHODS). In our study, $82 \%$ of cells had an OI $>0.7$, signifying strong selectivity, a proportion remarkably similar to the fraction qualitatively judged by ear to be orientation selective at the time of recording. Values $>1$ were common and occurred when there was inhibition of the spike rate to a level below baseline by the orthogonally oriented stimulus.

Qualitatively, 25\% of our V3 cell population were judged to be direction selective. Previous reports have found proportions ranging from $15 \%$ (qualitatively judged) (Baizer 1982) to $57 \%$ (quantitatively judged) (Gegenfurtner et al. 1997) of directionselective or biased cells in V3. When we calculated direction indices (DI) for these cells, 25\% were classified as direction selective and $19 \%$ direction biased. OI versus DI was plotted for the V3 cell population in Fig. 2.

\section{Color properties}

We routinely tested cells in V3 for color properties by presenting a series of roughly isoluminant bars of various colors on a black background at the cell's preferred orientation, size and speed. Very few cells (5\%) appeared to modulate their responses with wavelength as judged qualitatively at the time of recording. The few that did have wavelength preferences were often characterized by a lack of response to, rather than selectivity for, a particular wavelength. Cells of this nature were nevertheless classified as color-selective. When color indices were calculated for our population of V3 cells the 
proportion of selective cells was $9 \%$ and that of biased cells was $6 \%$.

\section{Disparity selectivity}

The primary focus of this study was the organization of disparity-selective cells in V3. To investigate their arrangement, long electrode penetrations were made at differing angles through V3d. Disparity-selective cells were classified into the groups first defined by Poggio and Fischer (1977). The following criteria were used for classification of disparityselective cells:

ASYMMETRIC CELLS. Excitatory responses greater than the sum of the monocular responses to a broad range of disparities either in front of the fixation plane (for near cells) or behind it (for far cells), commonly accompanied by inhibition, or a response smaller in magnitude than either monocular response, to the inverse range of disparities.

TUNED CELLS. A narrow $\left(<1^{\circ}\right.$ half-width at half height) peak in response occurring either at zero disparity (for T0 cells) or at near/far disparities for TN and TF cells. TI cells were characterized by excitation to all disparities except a narrow range $\left(<1^{\circ}\right.$ half-width at half height) of inhibition/response smaller than either of the monocular responses. Figure 3 shows the spike histograms and tuning curves for two typical disparity-selective cells in V3. The foveal separation during the recording of these cells was $1^{\circ}$, so we estimate the fixation plane to lie at $1^{\circ}$ on the $x$ axis. The upper cell was classified as a near cell, and the lower one, a T0 cell. Of our sample of 100 V3d cells tested for disparity, 61 were judged as disparityselective using the preceding criterion.

Disparity-tuning curves were plotted for each cell so that

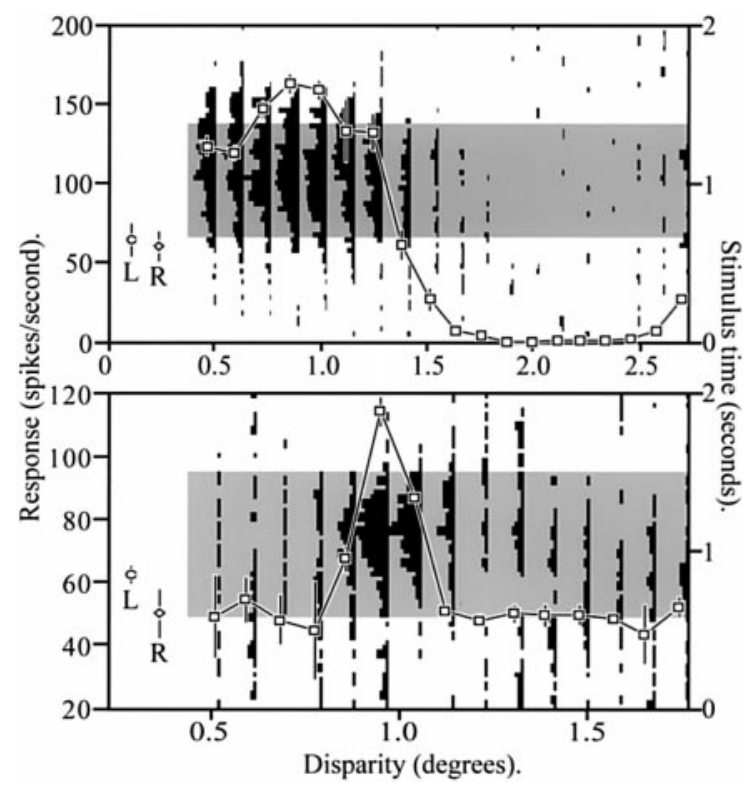

FIG. 3. Spike histograms and (superimposed) disparity-tuning curves for a near cell (top) and a tuned zero (T0) cell (bottom) recorded in V3d. $\square$, the time during which the disparity stimuli swept through the receptive fields of the cell. Bin width is $50 \mathrm{~ms}$. Mean monocular responses for the left and the right eye are labeled L and R. Error bars on the disparity-tuning curves are the standard errors of the mean spike rates from 4 stimulus presentations. The $x$ axis denotes the stimulus separation. The position of the fixation plane (foveal separation) was measured to be at $1^{\circ}$. they could be classified into one of the six groups outlined in the preceding text. Cell classification was sometimes difficult because the location of the fixation plane was only accurate to within $\pm 1.0^{\circ}$. However, it was possible to classify some cells (especially asymmetric cells) on the shape of their disparity tuning curves alone. Among tuned cells [tuned excitatory (T0), tuned near (TN), and tuned far (TF)], where accurate knowledge of the position of the fixation plane may affect the classification decision, other distinguishing features were used that aided classification: TE cells often had symmetrical inhibitory side flanks, whereas TN and TF cells often had longer asymmetric inhibitory regions on the side of the peak closer to zero disparity. Even if it was not always possible to distinguish among T0, TN, and TF cells, they could always be classified within the four groups: tuned (TO, TN, and TF), near, far, or unselective.

All types of disparity-selective cells were found in V3; the near cell was the most common while tuned inhibitory (TI), $\mathrm{TN}$, and TF cells were the most rare. Table 1 shows the percentages of disparity-selective cell types among a sample of 100 units in V3.

\section{Representative penetrations}

Three representative electrode penetrations will be presented. The first (Fig. 4A) passed through the cortical sheet at an angle of $44^{\circ}$ from normal; the second (Fig. $4 B$ ) traversed the cortical sheet at $73^{\circ}$. The third penetration (Fig. 5) passed through the cortex at the fundus of the lunate sulcus and therefore started normally, became tangential, and then exited the cortex normally again. Because this penetration changed angle as it passed through the cortex, it was divided into three equal segments, $a-c$. The average angle of each segment from normal was measured as 53,90 , and $36^{\circ}$.

In the first penetration (Fig. $4 A$ ), orientation preference changed by $36^{\circ}$ in a single step between two consecutive recording sites $100 \mu \mathrm{m}$ apart. All cells recorded in this penetration were near cells except the last one, which was unselective for disparity. In the second penetration (Fig. 4B) the orientation preference changed between consecutive cells in a progressive manner and covered $90^{\circ}$. Five separate types of disparity-selective cells were present along the length of the penetration, some in clusters.

In the third penetration (Fig. 5), orientation preference changed little at the beginning and the end of the passage through V3 where the electrode ran nearly perpendicular to the cortical layers. In the middle of the electrode track where the electrode was passing more tangentially, orientation preference changed most rapidly, covering a $90^{\circ}$ range over $1 \mathrm{~mm}$. The spans of disparity-selective cells changed in a similar way: long clusters were observed at the beginning and end of the penetration, whereas in the middle, the cell properties changed frequently. The clustering pattern of orientation selectivity properties in these electrode tracks confirms previous reports of a columnar organization of orientation-selective cells in V3 (Zeki 1978a). Additionally, disparity-selective cells also appear to be organized into columns that stretch from the cortical surface to white matter boundary.

The progression of receptive field $(\mathrm{RF})$ centers for each of these penetrations is shown in Fig. 6, $A-C$. The first penetration's RF centers were more clustered than those of the second 
TABLE 1. Proportions of disparity-selective cells found in V3d

\begin{tabular}{ccccccc}
\hline \hline T0, \% & TI, \% & TN, \% & TF, \% & Near, \% & Far, \% & Unselective, \% \\
\hline 15 & 4 & 4 & 5 & 22 & 11 & 39 \\
\hline
\end{tabular}

and third penetration illustrated. They also showed less net displacement. The RF centers in penetrations $(B)$ and $(C)$ progressed in an average downward direction because the electrode was advancing from posterior V3d, where the horizontal meridian is represented, toward the $\mathrm{V} 3 / \mathrm{V} 3 \mathrm{~A}$ border where the lower vertical meridian lies. The large amount of scatter in these progressions compared with that observed in similar penetrations through $\mathrm{V} 1$, reflects the coarse point-topoint retinotopy in $\mathrm{V} 3$.

\section{Columnar organization}

To distinguish between the clustering of cells with similar properties and the organization of cells into columns that stretch from the cortical surface to white matter boundary, we made penetrations through $\mathrm{V} 3$ over a range of angles to the cortical layers. If cells were organized into spherical clusters, the penetration angle would not affect the frequency with which different cell types were encountered. However, if cells were organized in a columnar fashion, one would encounter fewer shifts in disparity cell type for normal rather than tangential penetrations. To quantify this parameter, one first has to calculate the penetration angle. The electrode was always advanced close to the plane of section (horizontal), but the cortical sheet was rarely perfectly vertical. Thus measuring the horizontal angle of the electrode from the cortical layers in horizontal sections was not sufficient to describe the penetration angle in three dimensions. To overcome this geometrical problem, we used the following method to calculate the penetration angle. We first measured the length of the electrode track within V3. If one assumes an average cortical thickness of $1.8 \mathrm{~mm}$ these two lengths form two sides of a right-angle triangle. With this information, we calculated the penetration angle and the distance traversed parallel to the surface of the cortex for each penetration. We then counted the number of switches from one disparity tuned cell type to another that occurred over this tangential distance. The result was an index expressed as the number of cell type changes per mm measured parallel to the cortical surface. This parameter is plotted against penetration angle in Fig. 7. The positive correlation between these measures suggests an organization of disparity tuned cells into cortical columns rather than the simple clustering of cell properties.

\section{I S C U S S I O N}

Previous physiological investigations of $\mathrm{V} 3$ have shown it to be an area containing a predominance of orientation-selective cells, a subset of which are additionally direction-selective
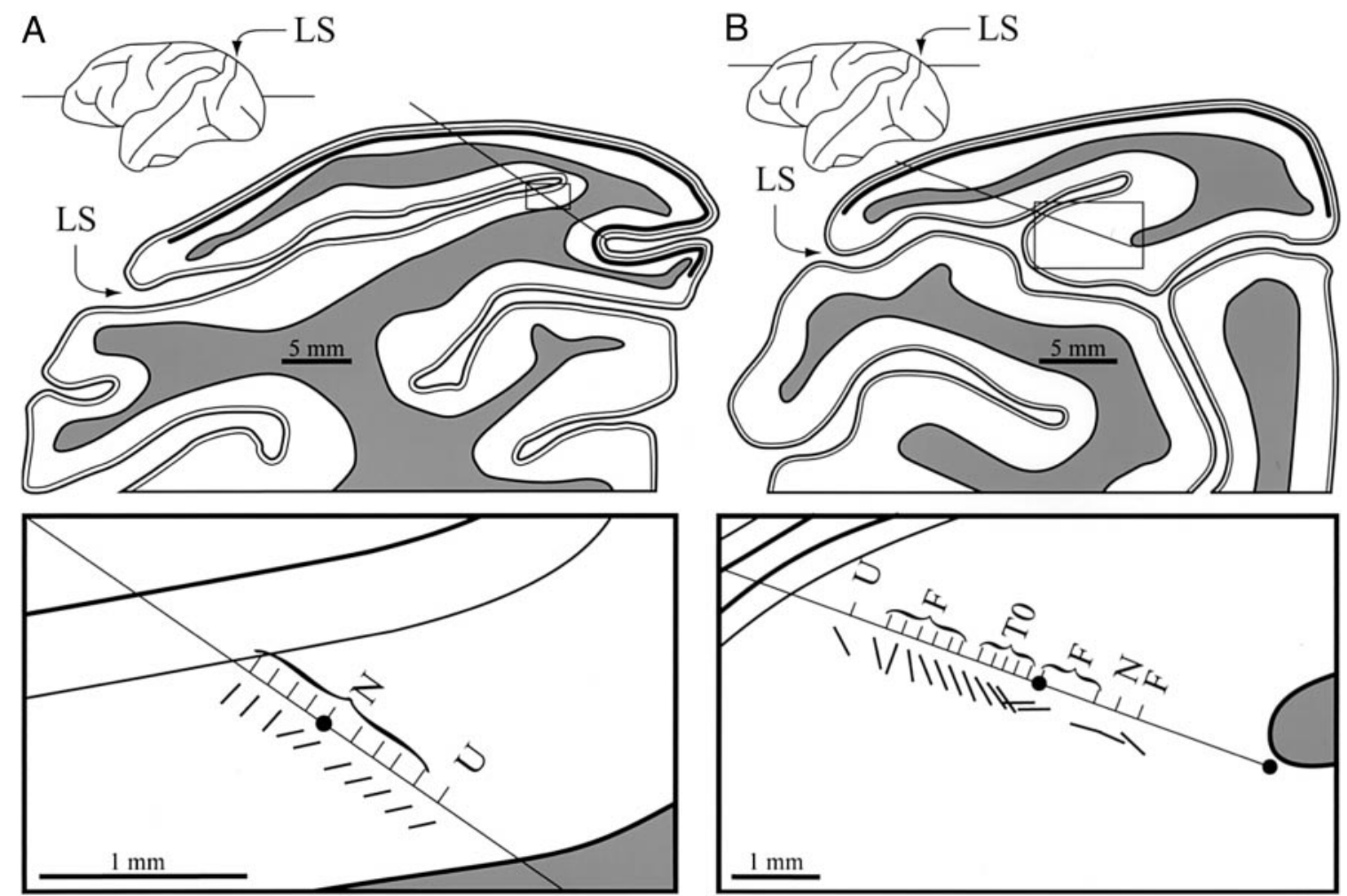

FIG. 4. Reconstructions of 2 electrode penetrations used to illustrate the functional organization of V3. Two horizontal sections are shown at the dorsoventral level indicated. A portion of the electrode track in V3d is boxed and shown at greater magnification. The position of each sampled cell is shown as a short line perpendicular to the electrode track. The preferred orientation of each cell is illustrated by an angled bar alongside the cell's position. A black dot on the electrode track indicates the position of an electrolytic lesion made in the cortex. The stria of Gennari is represented as a heavy line through the cortex. N, near; F, far; T0, tuned excitatory; TI, tuned inhibitory; TN, tuned near; TF, tuned far; U, unselective for disparity; LS, lunate sulcus. 

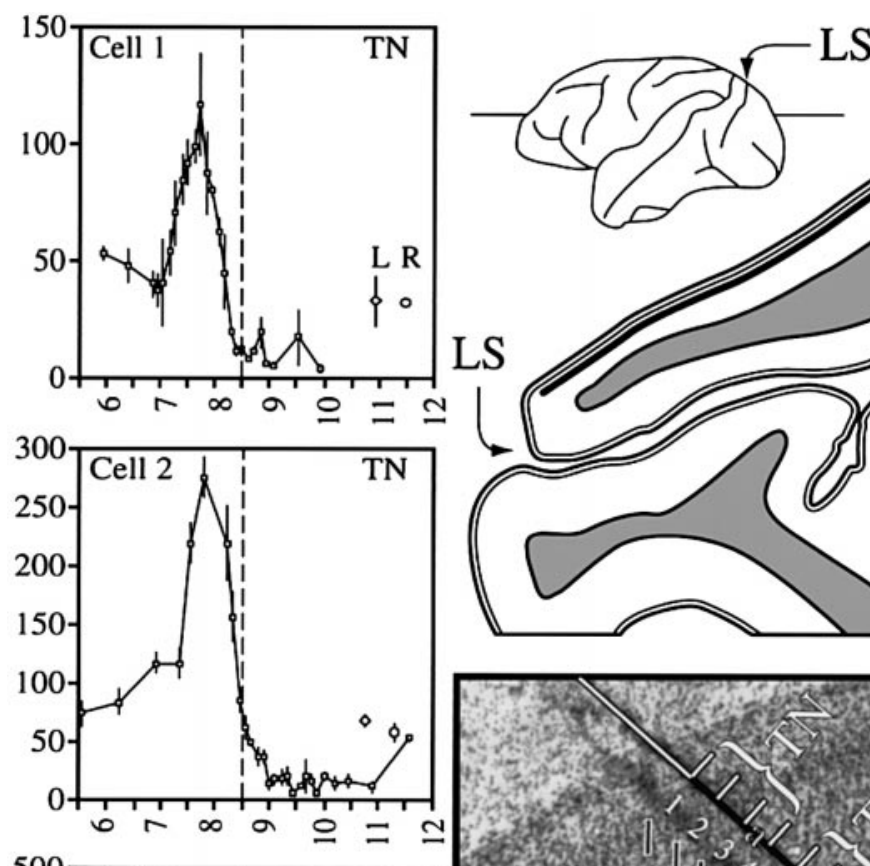

LS
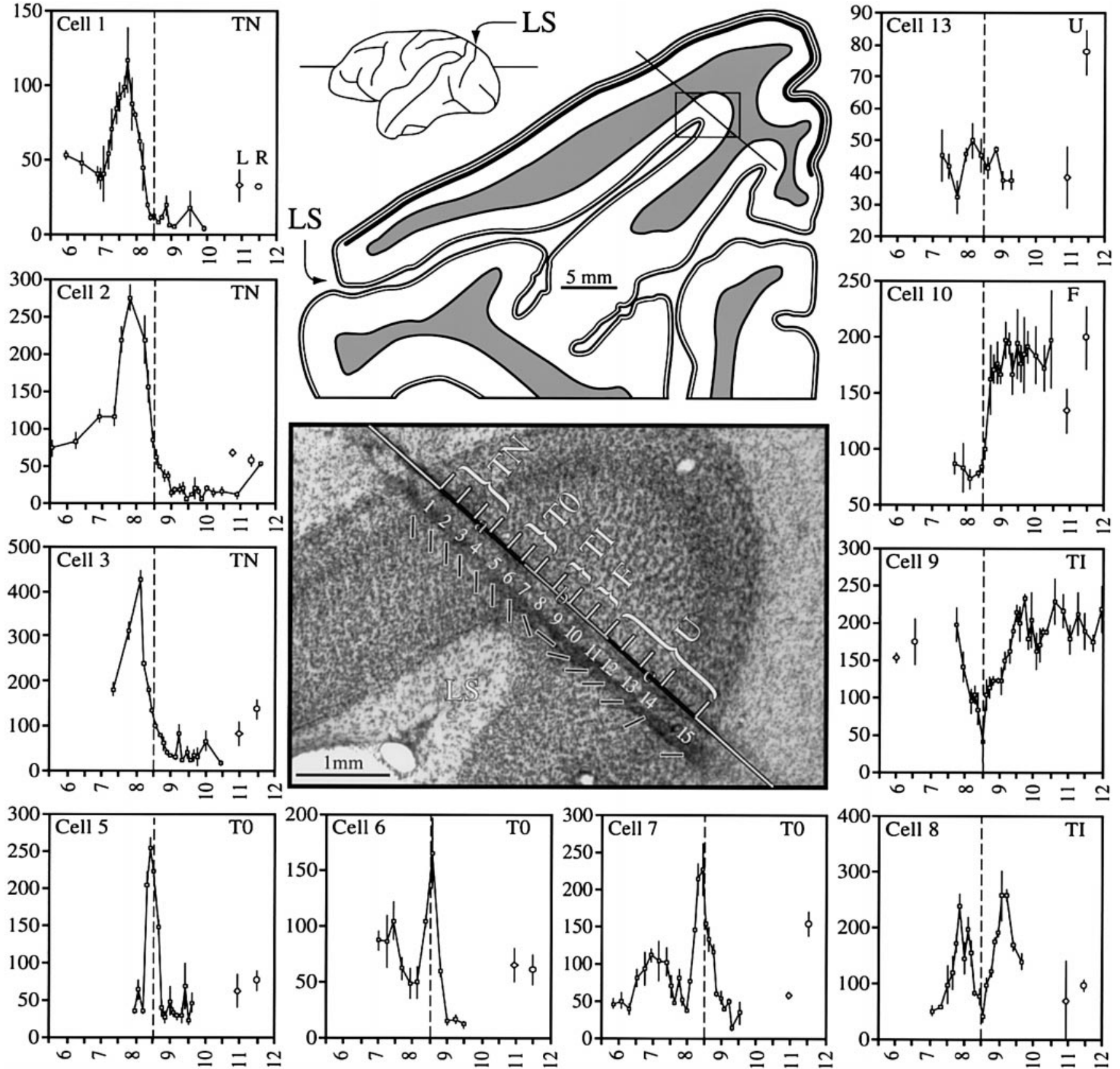

FIG. 5. Reconstruction of an electrode penetration that passed through the fundus of the lunate sulcus at the dorso-ventral level indicated. The Nissl-stained section shows the electrode track at greater magnification, with the position of each cell and its preferred orientation- and disparity-tuning category, as in Fig. 4. Cells are numbered and a disparity-tuning curve shown for a representative selection. Disparity-tuning curves are plotted as disparity (degrees of visual angle) on the X axis and response (mean spikes per second) on the $\mathrm{Y}$ axis. Error bars are standard errors of the mean of 6 trials. The position of the fixation plane (dotted line) was calculated at $8.5^{\circ} \pm 1.0^{\circ}$ this being the separation of the foveal projections on the tangent screen and the estimated error in projecting them with a reversible ophthalmoscope. Monocular responses are shown as single points with standard error bars: $\odot=$ Left eye monocular response, $\diamond=$ Right eye monocular response.

(Baizer 1982; Felleman and Van Essen 1987; Gegenfurtner et al. 1997; Zeki 1978a). Our present results are in agreement. On the basis of V3's complement of orientation-selective cells, its functional organization and preference for stimuli in motion, it has been proposed that V3 is an area specialized for the processing of dynamic form (Gegenfurtner et al. 1997; Zeki and Shipp 1988). The issue of whether color is processed within V3 is more contentious. Felleman and Van Essen (1987) found that $20 \%$ of V3 cells were color selective when judged by their quantitative color index criterion. Our results are not in agreement with those of Gegenfurtner et al. (1997), who found that $50 \%$ of V3 cells exhibited color selectivity as judged by an index-based method. Using the same quantitative method, about which we have reservations, we found only $15 \%$ of cells to be color selective, and when qualitatively judged at the time of recording, we categorized only $5 \%$ as color selective or 


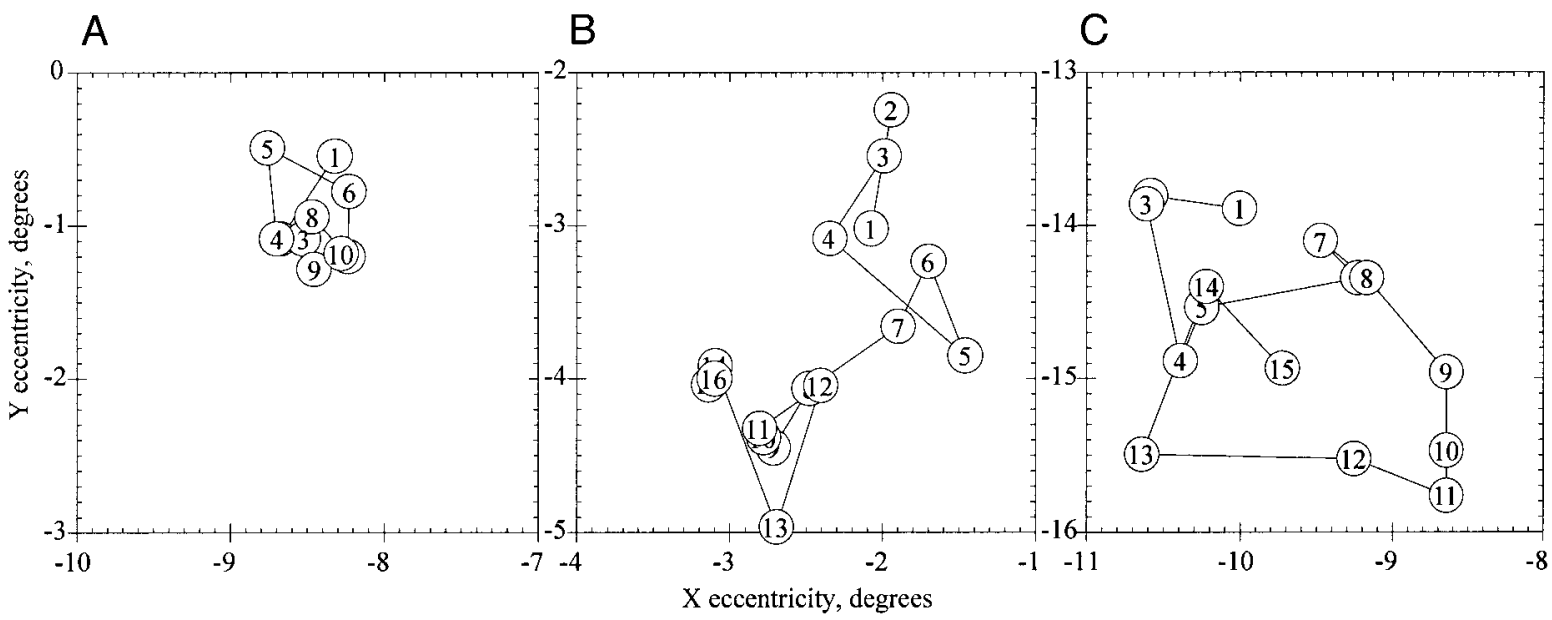

FIG. 6. Progression of receptive field (RF) centers of consecutive V3d cells in the 3 illustrated penetrations of Figs. 4 and 5. A: $\mathrm{RF}$ centers in this penetration were closely spaced with little net displacement. $B$ : compared with that of $A$, the RF centers of this penetration were more widely spaced and there was a net progression away from the horizontal meridian, as the electrode traveled away from the V3/V3 border (horizontal meridian) and toward the V3/V3A border (the lower vertical meridian). $C$ : a similar progression of RF centers to that shown in $B$. In both $B$ and $C$, there was a large amount of scatter, i.e., RF centers did not always advance in a perfectly ordered manner but followed a "random walk" with a gradual position change. This was due to the coarse point-to-point representation of the visual fields in V3.

biased. We conclude, as previously, that V3 is not involved in color processing.

Our finding that $61 \%$ of V3 cells were disparity selective strongly suggests that V3 is an area involved in the processing of disparity information. Moreover, our discovery of columns of cells with similar disparity-tuning properties demonstrates a functional specialization within V3 for stereoscopic depth. While the exact function of disparity columns has yet to be elucidated, it is likely that this functional architecture is important for simplifying both inter- and intra-areal connections between disparity-selective cells. Connections between disparity-selective cells are presumably important for the generation of cells with more sophisticated response properties, for example, selectivity for absolute distance using additional informa-

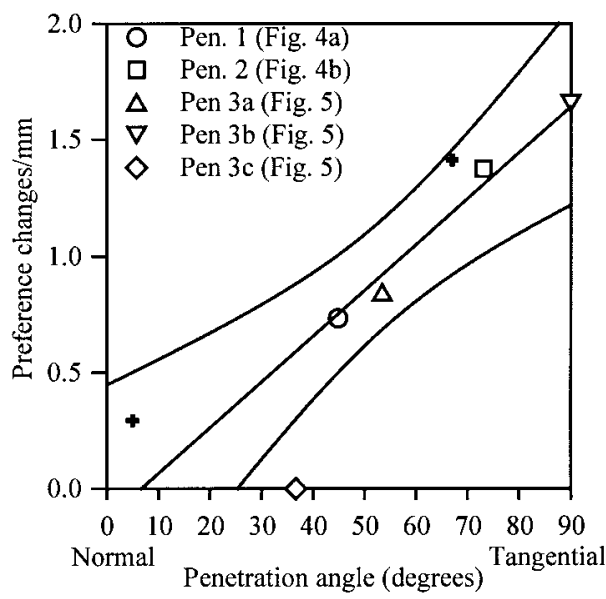

FIG. 7. Plot of penetration angle (degrees) vs. number of disparity cell types changes per millimeter. The three penetrations illustrated in Figs. 4 and 5 are indicated. Because the penetration of Fig. 5 changes angle as it passed through the cortex, it was split into 3 equal segments $(\mathrm{a}-\mathrm{c})$. The average angle and number of cell changes $/ \mathrm{mm}$ were calculated separately for each segment. Un-illustrated penetrations are shown as crosses. A linear regression is fitted to the data with $95 \%$ confidence intervals. The positive correlation indicates the presence of cortical columns. tion about eye position or selectivity for disparity gradients using the outputs from many disparity-selective cells.

DeAngelis et al. (DeAngelis and Newsome 1999) have recently reported finding a similar organization of disparity columns in visual area V5 (MT) of the macaque. The columnar organization we describe bears a resemblance to their V5 disparity columns except that the cells described here are orientation/disparity selective while those in V5 are direction/ disparity selective. They also demonstrated that microstimulation of these columns influenced the depth judgements of macaques (DeAngelis et al. 1998). They concluded that V5 plays an important role in stereoscopic depth perception in addition to its well known role in motion perception. It is very likely that cells in V5 tuned for direction of motion and disparity are signaling the depth of moving objects. V5 cells capable of signaling motion in depth have also been found (Zeki 1974b; but see Maunsell and Van Essen 1983). Since V3 appears to be an area specialized for form vision, its high proportion and functional organization of disparity tuned cells would suggest that cells there are signaling three-dimensional form. It would therefore be interesting to discover if the processing of 3D information in V3 could be correlated with perception of depth in the same way that has been demonstrated in V5 or whether the disparity-selective cells of V3 are serving a "nonconscious" function, such as the guidance of vergence eye movements (Cumming and Parker 1997; Masson et al. 1997).

We found a higher proportion of near cells than any other type of disparity-selective cell in V3. Previous studies of disparity tuning in prestriate cortex have found that there is an increase in the ratio of asymmetric to tuned cells as one advances from V1-V5 to V5A (MST) and LIP (Gnadt and Mays 1991; Maunsell and Van Essen 1983; Poggio et al. 1988; Roy et al. 1992; Trotter 1995; Trotter et al. 1992). The functional significance of this proportional change is not yet fully understood because the functions of each type of disparity tuned cell is not known. It was originally suggested that tuned 
cells (T0, TN, and TF) might have a role in fine stereoscopic discrimination around the fixation plane, whereas asymmetric cells might participate in coarse stereoscopic discrimination necessary to drive vergence eye movements (Poggio and Fischer 1977). However, it has since been demonstrated that cells with asymmetric disparity tuning can be better suited to providing fine stereoscopic information than those with symmetric tuning (Lehky et al. 1990). Other recent studies have questioned the classification scheme of Poggio and Fischer altogether, suggesting that there is in fact a smooth distribution of disparity tuning curve shape and preference (DeAngelis and Newsome 1999; LeVay and Voigt 1988). Most of the disparity tuned cells recorded in this study were readily classifiable into the groups defined by Poggio et al. (Poggio and Fischer 1977; Poggio et al. 1988) but it must be noted that this classification was not always straightforward, and no bimodal distribution of width of tuning was seen. Instead we chose a somewhat arbitrary threshold of $1^{\circ}$ half-width at half-height to distinguish symmetric and asymmetric cells, and our data are not incompatible with the idea that disparity tuning forms a continuum of shapes and preferences.

Recently V3's connections with posterior parietal areas involved in spatial vision have been demonstrated. V3 sends ascending connections to V6 (PO) (Shipp et al. 1998), an area in the anterior bank of the parieto-occipital sulcus heavily involved in sensory motor integration of reaching arm movements (Galletti et al. 1997). V6 also contains a population of "real position" cells, thought to be engaged in the spatial encoding of extrapersonal visual space (Galletti et al. 1995). These cellular properties would require, or at least benefit from, stereoscopic depth information. In a concurrent study (Adams 1997), we have found connections from V3 (via V3A) to an area at the caudal extent of the intraparietal sulcus (CIP) (Kusunoki et al. 1993). CIP contains cells that use disparity information to code the axis- and surface-orientation and shape of objects in three dimensions (Endo et al. 2000; Sakata et al. 1997). We suggest that the disparity-selective cells of V3 provide at least some of the information required for the generation of more complex responses to three-dimensional forms found in CIP cells.

We thank Drs. Stuart Shipp and Konstantinos Moutoussis for participating in the experiments, J. Romaya for stimulus and data collection programming, G. Wray for technical assistance, and Drs. Jonathan Horton and Lawrence Sincich and an anonymous referee for helpful comments on the manuscript.

This work was supported by the Wellcome Trust.

\section{REFERENCES}

Adams DL. Functional Organization of the Monkey Visual Cortex for Stereoscopic Depth ( $\mathrm{PhD}$ thesis). London: University College London, 1997.

Albright TD, Desimone R, AND GRoss CG. Columnar organization of directionally selective cells in visual area MT of the macaque. J Neurophysiol 51: $16-31,1984$.

Andersen RA, Asanuma C, Essick G, AND Siegel RM. Corticocortical connections of anatomically and physiologically defined subdivisions within the inferior parietal lobule. J Comp Neurol 296: 65-113, 1990.

BAIZER JS. Receptive field properties of V3 neurons in monkey. Invest Ophthalmol Vis Sci 23: 87-95, 1982.

Blatt GJ, ANDERSEn RA, AND Stoner GR. Visual receptive field organization and cortico-cortical connections of the lateral intraparietal area (area LIP) in the macaque. J Comp Neurol 299: 421-445, 1990.
Burkhalter A, Felleman DJ, Newsome WT, and Van Essen DC. Anatomical and physiological asymmetries related to visual areas V3 and VP in macaque extrastriate cortex. Vision Res 26: 63-80, 1986.

CRAGG BG. The topography of the afferent projections in the circumstriate visual cortex of the monkey studied by the Nauta method. Vision Res 9: 733-747, 1969.

CUMming BG AND PARKER AJ. Responses of primary visual cortical neurons to binocular disparity without depth perception. Nature 389: 280-283, 1997.

DeAngelis GC, Cumming BG, And Newsome WT. Cortical area MT and the perception of stereoscopic depth. Nature 394: 677-680, 1998.

DeAngelis GC and Newsome WT. Organization of disparity-selective neurons in macaque area MT. J Neurosci 19: 1398-1415, 1999.

Endo K, HaranaKa Y, Shein WN, Adams DL, Kusunoki M, and SaKata H. Effects of different types of disparity cues on the response of axis-orientation selective cells in the monkey parietal cortex. Nippon Ganka Gakkai Zasshi 104: 334-343, 2000.

Felleman DJ AND VAN Essen DC. Receptive field properties of neurons in area V3 of macaque monkey extrastriate cortex. J Neurophysiol 57: 889920, 1987.

Galletti C, Battaglini PP, and Fattori P. Eye position influence on the parieto-occipital area PO (V6) of the macaque monkey. Eur J Neurosci 7: 2486-2501, 1995.

Galletti C, Fattori P, Kutz DF, and Battaglini PP. Arm movementrelated neurons in the visual area V6A of the macaque superior parietal lobule. Eur J Neurosci 9: 410-413, 1997.

GaLlyas F. Silver staining of myelin by means of physical development. Neurol Res 1: 203-209, 1979.

GatTass R, Sousa AP, AND Gross CG. Visuotopic organization and extent of V3 and V4 of the macaque. J Neurosci 8: 1831-1845, 1988.

Gegenfurtner KR, KIPER DC, AND LevitT JB. Functional properties of neurons in macaque area V3. J Neurophysiol 77: 1906-1923, 1997.

GNADT JW AND MAYS LE. Depth-tuning in area LIP by disparity and accommodative cues. Soc Neurosci Abstr 17: 443-511, 1991.

HENRY GH, Bishop PO, AND COOMBS JS. Inhibitory and sub-liminal excitatory receptive fields of simple units in cat striate cortex. Vision Res 9: 12891296, 1969.

Hubel DH AND WIESEL TN. Stereoscopic vision in macaque monkey. Cells sensitive to binocular depth in area 18 of the macaque monkey cortex. Nature 225: 41-42, 1970.

Kusunoki M, Tanaka Y, Ohtsuka K, and Sakata H. Selectivity of the parietal visual neurons in the axis orientation of objects in space. Soc Neurosci Abstr 19: 770, 1993.

LehKy SR, Pouget A, AND SeJNOwSKI TJ. Neural models of binocular depth perception. Cold Spring Harbor Symp Quant Biol 55: 765-777, 1990.

LeVAy S AND VoIGT T. Ocular dominance and disparity coding in cat visual cortex. Visual Neurosci 1: 395-414, 1988.

Lund JS, Lund RD, Hendrickson AE, Bunt AH, AND Fuchs AF. The origin of efferent pathways from the primary visual cortex, area 17, of the macaque monkey as shown by retrograde transport of horseradish peroxidase. J Comp Neurol 164: 287-303, 1975.

LYON DC AND KAAS JH. Connectional and architectonic evidence for dorsal and ventral V3, and dorsomedial area in marmoset monkeys. J Neurosci 21 : 249-261, 2001.

Masson GS, Busettini C, And Miles FA. Vergence eye movements in response to binocular disparity without depth perception. Nature 389: $283-$ 286, 1997.

Maunsell JH and Van Essen DC. Functional properties of neurons in middle temporal visual area of the macaque monkey. II. Binocular interactions and sensitivity to binocular disparity. J Neurophysiol 49: 1148-1167, 1983.

Merrill EG AND Ainsworth A. Glass-coated platinum-plated tungsten microelectrodes. Med Biol Eng 10: 662-672, 1972.

MountCASTLE VB. The columnar organization of the neocortex. Brain 120: 701-722, 1997.

Perkel DJ, Bullier J, ANd Kennedy H. Topography of the afferent connectivity of area 17 in the macaque monkey: a double-labelling study. J Comp Neurol 253: 374-402, 1986.

POGGIO GF AND Fischer B. Binocular interaction and depth sensitivity in striate and prestriate cortex of behaving rhesus monkey. J Neurophysiol 40 : 1392-1405, 1977. 
Poggio GF, Gonzalez F, And Krause F. Stereoscopic mechanisms in monkey visual cortex: binocular correlation and disparity selectivity. J Neurosci 8: 4531-4550, 1988.

Roy JP, Komatsu H, AND Wurtz RH. Disparity sensitivity of neurons in monkey extrastriate area MST. J Neurosci 12: 2478-2492, 1992.

Sakata H, Taira M, Kusunoki M, Murata A, and Tanaka Y. The TINS Lecture. The parietal association cortex in depth perception and visual control of hand action. Trends Neurosci 20: 350-357, 1997.

ShipP S, Blanton M, AND ZEKI S. A visuo-somatomotor pathway through superior parietal cortex in the macaque monkey: cortical connections of areas V6 and V6A. Eur J Neurosci 10: 3171-3193, 1998.

Tootell RB, Mendola JD, Hadjikhani NK, Liu AK, and Dale AM. The representation of the ipsilateral visual field in human cerebral cortex. Proc Nat Acad Sci USA 95: 818-824, 1998.

Trotter Y. Cortical representation of visual three-dimensional space. Perception 24: 287-298, 1995.

Trotter Y, Celebrini S, Stricanne B, Thorpe S, and Imbert M. Modulation of neural stereoscopic processing in primate area V1 by the viewing distance. Science 257: 1279-1281, 1992.

VAN ESSEN DC AND ZEKI SM. The topographic organization of rhesus monkey prestriate cortex. J Physiol (Lond) 277: 193-226, 1978.

WhitTERIDGE D. Area 18 and the vertical meridian of vision. In: Functions of the Corpus Callosum, edited by Ettlinger EG. London: Churchill, 1965, p. 115.

WONG-RILEY MTT. Changes in the visual system of monocularly sutured or enucleated cats demonstrable with cytochrome oxidase histochemistry. Brain Res 171: 11-28, 1979.

ZEKI SM. Representation of central visual fields in prestriate cortex of monkey. Brain Res 14: 271-291, 1969.

ZEKI SM. Functional organization of a visual area in the posterior bank of the superior temporal sulcus of the rhesus monkey. J Physiol (Lond) 236: 549-573, 1974a.

ZEKI SM. Cells responding to changing image size and disparity in the cortex of the rhesus monkey. J Physiol (Lond) 242: 827-841, 1974 b.

ZEKI SM. The third visual complex of rhesus monkey prestriate cortex. J Physiol (Lond) 277: 245-272, 1978a.

ZEKI SM. The cortical projections of foveal striate cortex in the rhesus monkey. J Physiol (Lond) 277: 227-244, 1978b.

ZEKI S. A direct projection from area V1 to area V3A of rhesus monkey visual cortex. Proc R Soc Lond B Biol Sci 207: 499-506, 1980.

ZEKI SM AND SANDEMAN DR. Combined anatomical and electrophysiological studies on the boundary between the second and third visual areas of rhesus monkey cortex. Proc Roy Soc Lond B Biol Sci 194: 555-562, 1976.

ZEKI S AND SHIPP S. The functional logic of cortical connections. Nature 335: 311-317, 1988 . 